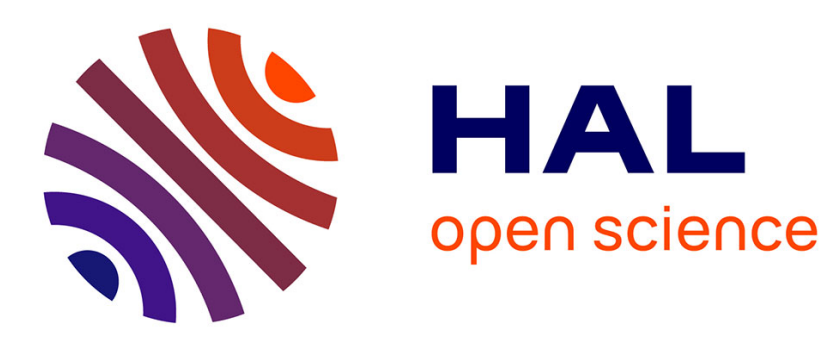

\title{
Degree and class of caustics by reflection for a generic source
}

\author{
Alfrederic Josse, Françoise Pene
}

\section{To cite this version:}

Alfrederic Josse, Françoise Pene. Degree and class of caustics by reflection for a generic source. 2013. hal-00771782

\section{HAL Id: hal-00771782 \\ https://hal.science/hal-00771782}

Preprint submitted on 9 Jan 2013

HAL is a multi-disciplinary open access archive for the deposit and dissemination of scientific research documents, whether they are published or not. The documents may come from teaching and research institutions in France or abroad, or from public or private research centers.
L'archive ouverte pluridisciplinaire HAL, est destinée au dépôt et à la diffusion de documents scientifiques de niveau recherche, publiés ou non, émanant des établissements d'enseignement et de recherche français ou étrangers, des laboratoires publics ou privés. 


\title{
DEGREE AND CLASS OF CAUSTICS BY REFLECTION FOR A GENERIC SOURCE
}

\author{
ALFREDERIC JOSSE AND FRANÇOISE PÈNE
}

\begin{abstract}
Given any irreducible algebraic (mirror) curve $\mathcal{C} \subseteq \mathbb{P}^{2}:=$ $\mathbb{P}^{2}(\mathbb{C})$ and any (light position) $S \in \mathbb{P}^{2}$, the caustic by reflection $\Sigma_{S}(\mathcal{C})$ of $\mathcal{C}$ from $S$ is the Zariski closure of the envelope of the reflected lines got from the lines coming from $S$ after reflection on $\mathcal{C}$. In $[7,8]$, we established formulas for the degree and class (with multiplicity) of $\Sigma_{S}(\mathcal{C})$ for any $\mathcal{C}$ and any $S$. In this paper, we prove the birationality of the caustic map for a generic $S$ in $\mathbb{P}^{2}$. Moreover, we give simple formulas for the degree and class (without multiplicity) of $\Sigma_{S}(\mathcal{C})$ for any $\mathcal{C}$ and for a generic $S$ in $\mathbb{P}^{2}$.
\end{abstract}

\section{INTRODUCTION}

We are interested in the study of caustics by reflection in the projective complex plane $\mathbb{P}^{2}$. Given an irreducible algebraic curve $\mathcal{C}=V(F) \subset \mathbb{P}^{2}$ of degree $d \geq 2$ and given $S=\left[x_{0}: y_{0}: z_{0}\right] \in \mathbb{P}^{2}$, the caustic by reflection $\Sigma_{S}(\mathcal{C})$ of $\mathcal{C}$ from $S$ is the Zariski closure of the envelope of the reflected lines on $\mathcal{C}$ of the lines coming from $S$.

For $m \in \mathcal{C}$, the reflected line $\mathcal{R}_{m, S, \mathcal{C}}$ is defined as the orthogonal symmetric of the (incident) line $(m S)$ with respect to the tangent line to $\mathcal{C}$ at $m$. In $[7$, 8], we detail the construction of the reflected lines and we define two rational maps $\rho_{F, S}$ and $\Phi_{F, S}$ from $\mathbb{P}^{2}$ into itself satisfying the following property: For a generic $m$ in $\mathcal{C}, \rho_{F, S}(m)$ corresponds to an equation of the reflected line $\mathcal{R}_{m, S, \mathcal{C}}$ and this line is tangent to $\Phi_{F, S}(\mathcal{C})$ at $\Phi_{F, S}(m)$. Hence the caustic $\Sigma_{S}(\mathcal{C})$ is the Zariski closure of $\Phi_{F, S}(\mathcal{C})$ and $\Phi_{F, S}$ is called the caustic map of $\mathcal{C}$ from $S$. Observe that the Zariski closure of $\rho_{F, S}(\mathcal{C})$ is then the dual curve of the caustic $\Sigma_{S}(\mathcal{C})$. In [7,8], we used this approach to establish precise formulas for the degree anf class (both with multiplicity) of $\Sigma_{S}(\mathcal{C})$ for any $\mathcal{C}$ and any $S$. The degree with multiplicity of $\Sigma_{S}(\mathcal{C})$ means its degree multiplied by the degree of the rational map $\Phi_{F, S}$ restricted to $\mathcal{C}$ . The class with multiplicity of $\Sigma_{\mathcal{S}}(\mathcal{C})$ means its class multiplied by the degree of the rational map $\rho_{F, S}$ restricted to $\mathcal{C}$. Our formulas complete the formula obtained by Chasles in [3] for the class of a caustic by reflection (for a generic $\mathcal{C}$ and a generic $S$ ). Let us indicate that, in [1], Brocard and Lemoyne gave, without any proof, formulas for the degree and class of caustics by reflection (for a Plücker curve $\mathcal{C}$ and for $S$ not at infinity). It seems that their formulas come from an incorrect composition of formulas

Date: January 9, 2013.

2000 Mathematics Subject Classification. 14H50,14E05,14N05,14N10.

Key words and phrases. caustic, degree, class, birationality

Françoise Pène is supported by the french ANR project GEODE (ANR-10-JCJC-0108). 
by Salmon and Cayley [10] for some caracteristic invariants of pedal and evolute curves (using the representation of caustics by reflection given by Quetelet and Dandelin). This is discussed in [8]. Let us also mention the work of Catanese and Trifogli on focal loci, which generalize evolutes to higher dimension [11, 2].

The question of the birationality of the rational maps $\rho_{F, S}$ and $\Phi_{F, S}$ on $\mathcal{C}$ is not evident even if $S$ is not at infinity. Indeed, according to results of Quetelet and Dandelin [9, 4], when $S$ is not at infinity, the caustic $\Sigma_{S}(\mathcal{C})$ is the evolute of the $S$-centered homothety (with ratio 2 ) of the pedal of $\mathcal{C}$ from $S$ (i.e. the evolute of the orthotomic of $\mathcal{C}$ with respect to $S$ ). But we just know that the evolute map is birational for a generic algebraic curve (see [5] by Fantechi).

In this note, we prove the birationality on $\mathcal{C}$ of the maps $\rho_{F, S}$ and $\Phi_{F, S}$ for any irreducible algebraic curve $\mathcal{C} \subset \mathbb{P}^{2}$ of degree $d \geq 2$ and for a generic $S$ in $\mathbb{P}^{2}$. This result enables us to establish simple formulas for the degree and class of caustics by reflection valid for any irreducible algebraic curves $\mathcal{C} \subset \mathbb{P}^{2}$ of degree $d \geq 2$ and for a generic $S$ in $\mathbb{P}^{2}$. In this study, the cyclic points $I=[1: i: 0]$ and $J=[1:-i: 0]$ play a particular role. We will also use the canonical projection $\pi: \mathbb{C}^{3} \backslash\{0\} \rightarrow \mathbb{P}^{2}$.

\section{Birationality}

Theorem 2.1. Let $\mathcal{C}=V(F) \subset \mathbb{P}^{2}$ be any irreducible algebraic curve of degree $d \geq 2$. For a generic $S \in \mathbb{P}^{2}$, the maps $\rho_{F, S}$ and $\Phi_{F, S}$ are birational on $\mathcal{C}$.

Before going into the proof of our Theorem, let us introduce some notations and recall some facts (see [7]). For any line $\mathcal{D}=V(a x+b y+c z) \in \mathbb{P}^{2}$ such that $a^{2}+b^{2} \neq 0$, we define the orthogonal symmetric with respect to $\mathcal{D}$ as the rational map $\sigma_{\mathcal{D}}: \mathbb{P}^{2} \rightarrow \mathbb{P}^{2}$ (which is an involution) given by

$$
\sigma_{\mathcal{D}}[x: y: z]=\pi\left(\left(a^{2}+b^{2}\right) \cdot(x, y, z)+(a x+b y+c z) \cdot(a, b, 0)\right) .
$$

Let $\mathcal{C}=V(F) \subset \mathbb{P}^{2}$ be an irreducible algebraic curve of degree $d \geq 2$ and let $S \in \mathbb{P}^{2} \backslash\{I, J\}$ be a (light) position. We define $C_{0}:=\mathcal{C} \backslash V\left(F_{x}^{2}+F_{y}^{2}\right)$. Observe that this set corresponds to the complement in $\mathcal{C}$ of the cyclic apparent contour of $\mathcal{C}$ (the cyclic apparent contour of $\mathcal{C}$ from the cyclic points). We recall that the reflected line $\mathcal{R}_{m, S, \mathcal{C}}$ at $m \in \mathcal{C}_{0} \backslash\{S\}$ is the line $\left(m \sigma_{\mathcal{T}_{m} \mathcal{C}}(S)\right)$, where $\mathcal{T}_{m} \mathcal{C}$ is the tangent to $\mathcal{C}$ at $m$. For any $m \in \mathcal{C}_{0}$, we define the normal line $\mathcal{N}_{m} \mathcal{C}$ to $\mathcal{C}$ at $m$ as the line containing $m$ and $\left[F_{x}(m): F_{y}(m): 0\right]$.

For any $m \in \mathcal{C}_{0}$, we consider the set $K_{m}$ of points $S \in \mathbb{P}^{2}$ such that there exists $m^{\prime} \in \mathcal{C}_{0} \backslash\{m\}$ satisfying $\rho_{F, S}\left(m^{\prime}\right)=\rho_{F, S}(m) \neq 0$. Observe that the set $\mathcal{A}$ of $S \in \mathbb{P}^{2}$ such that $\rho_{F, S}$ is not birational can be written $\mathcal{A}=$ $\bigcup_{E \subset \mathcal{C}_{0}: \# E<\infty} \bigcap_{m \in \mathcal{C}_{0} \backslash E} K_{m}$. To prove that $\rho_{F, S}$ is birational for a generic $S$ in $\mathbb{P}^{2}$, we prove that $\mathcal{A}$ is contained in a subvariety of codimension at least 1 in $\mathbb{P}^{2}$. Our proof is based on the following lemma.

Lemma 2.2. For any $m \in \mathcal{C}_{0}$, the set $K_{m}$ is contained in a (possibly non irreducible) algebraic curve $\bar{K}_{m}$ of degree at most $2 d^{2}+2$.

Proof. Let us consider any $m \in \mathcal{C}_{0}$. Let $S \in K_{m}$ and $m^{\prime} \in \mathcal{C}_{0} \backslash\{m\}$ satisfying $\rho_{F, S}\left(m^{\prime}\right)=\rho_{F, S}(m) \neq 0$. Then $\left(m m^{\prime}\right)=\mathcal{R}_{m, S, \mathcal{C}}=\mathcal{R}_{m^{\prime}, S, \mathcal{C}}$ and so $S$ is in 
$\mathcal{A}_{m, m^{\prime}}:=\sigma_{\mathcal{T}_{m} \mathcal{C}}\left(\left(m m^{\prime}\right)\right) \cap \sigma_{\mathcal{T}_{m^{\prime}} \mathcal{C}}\left(\left(m m^{\prime}\right)\right)$. Observe that, if $\sigma_{\mathcal{T}_{m} \mathcal{C}}\left(\left(m m^{\prime}\right)\right)=$ $\sigma_{\mathcal{T}_{m^{\prime}} \mathcal{C}}\left(\left(m m^{\prime}\right)\right)$, then these lines are $\left(m m^{\prime}\right)$ and so $\left(m m^{\prime}\right)$ is stable by $\sigma_{\mathcal{T}_{m} \mathcal{C}}$ and by $\sigma_{\mathcal{T}_{m^{\prime}}} \mathcal{C}$. But $\mathcal{T}_{m} \mathcal{C}$ and $\mathcal{N}_{m} \mathcal{C}$ are the only lines containing $m$ which are stable by $\sigma_{\mathcal{T}_{m} \mathcal{C}}$. Therefore, $\sigma_{\mathcal{T}_{m} \mathcal{C}}\left(\left(m m^{\prime}\right)\right)=\sigma_{\mathcal{T}_{m^{\prime}} \mathcal{C}}\left(\left(m m^{\prime}\right)\right)$, implies that $\left(m m^{\prime}\right) \in\left\{\mathcal{T}_{m} \mathcal{C}, \mathcal{N}_{m} \mathcal{C}\right\} \cap\left\{\mathcal{T}_{m^{\prime}} \mathcal{C}, \mathcal{N}_{m^{\prime}} \mathcal{C}\right\}$. If $\left\{\mathcal{T}_{m} \mathcal{C}, \mathcal{N}_{m}^{\prime} \mathcal{C}\right\} \cap\left\{\mathcal{T}_{m^{\prime}} \mathcal{C}, \mathcal{N}_{m^{\prime}} \mathcal{C}\right\}=\emptyset$, then $S$ is the only point of $\mathcal{A}_{m, m^{\prime}}$, so $S$ is equal to

$$
\tau_{m}\left(m^{\prime}\right):=\pi\left(\left(m \wedge \sigma_{\mathcal{T}_{m} \mathcal{C}}\left(m^{\prime}\right)\right) \wedge\left(m^{\prime} \wedge \sigma_{\mathcal{T}_{m^{\prime}} \mathcal{C}}(m)\right)\right) .
$$

Notice that $\tau_{m}$ is a rational map with coordinates of degree $2 d$. We obtain that $S$ belongs to the Zariski closure of $\tau_{m}(\mathcal{C})$, which (according to [6, Proposition 4.4]) is contained in an algebraic curve of degree at most $\mathcal{C} \cdot \tau_{m}^{*}(H) \leq 2 d^{2}$ (where $H$ is the hyperplane class in $\mathbb{P}^{2}$ ). Otherwise, $S \in \mathcal{A}_{m, m^{\prime}}=\left(m m^{\prime}\right) \in\left\{\mathcal{T}_{m} \mathcal{C}, \mathcal{N}_{m} \mathcal{C}\right\}$. Finally, we have $K_{m} \subseteq \bar{K}_{m}:=$ $\overline{\tau_{m}(\mathcal{C})} \cup \mathcal{T}_{m} \mathcal{C} \cup \mathcal{N}_{m} \mathcal{C}$ which is an algebraic curve of degree at most $2 d^{2}+2$ (use for example the fundamental lemma of [7] applied with $\tau_{m}$ ).

Proof of Theorem 2.1. Let us prove that $\rho_{F, S}$ is birational on $\mathcal{C}$ for a generic $S$ in $\mathbb{P}^{2}$. The birationality of $\Phi_{F, S}$ will follow. Indeed, for a generic $S$ in $\mathbb{P}^{2}$, the caustic $\Sigma_{S}(\mathcal{C})$ is a curve (see for example [8]). Therefore, for generic $m, m^{\prime} \in \mathcal{C}, \Phi_{F, S}(m)=\Phi_{F, S}\left(m^{\prime}\right)$ implies that $\rho_{F, S}(m)=\rho_{F, S}\left(m^{\prime}\right)$. With the notations of Lemma 2.2 , we define $\mathcal{A}^{\prime}:=\bigcup_{E \subset \mathcal{C}_{0}: \# E<\infty} \bigcap_{m \in \mathcal{C}_{0} \backslash E} \bar{K}_{m}$. We prove that the set $\mathcal{F}:=\left\{\bigcap_{m \in \mathcal{C}_{0} \backslash E} \bar{K}_{m}, E \subset \mathcal{C}_{0}, \# E<\infty\right\}$ is inductive for the inclusion. Let $\left(\mathcal{F}_{j}:=\bigcap_{m \in \mathcal{C}_{0} \backslash E_{j}} \bar{K}_{m}\right)_{j>1}$ be an increasing sequence of sets belonging to $\mathcal{F}$. Let us show that the union $Z$ of these sets is also in $\mathcal{F}$. First $Z \subseteq \bigcap_{m \in \mathcal{C}_{0} \backslash \bigcup_{i>1} E_{i}} \bar{K}_{m} \subseteq \bar{K}_{m_{0}}$ for some fixed $m_{0} \in \mathcal{C}_{0} \backslash \bigcup_{i \geq 1} E_{i}$. Now $\bar{K}_{m_{0}}$ is the union of a finite number of irreducible algebraic curves $C_{1}, \ldots, C_{p}$. Let $i \in\{1, \ldots, p\}$ and let $d_{i}$ be the degree of $C_{i}$. If $C_{i} \subseteq Z$, then there exists $N_{i} \geq 1$ such that $C_{i} \subseteq \mathcal{F}_{N_{i}}$. Assume now that $C_{i} \not \subset Z$. Then $\left(C_{i} \cap \mathcal{F}_{j}\right)_{j \geq 1}$ is an increasing sequence of finite sets containing at most $d_{i} \times\left(2 d^{2}+2\right)$ points. Therefore, there exists $N_{i} \geq 1$ such that $\left(C_{i} \cap Z\right) \subseteq \mathcal{F}_{N_{i}}$. We conclude that $Z=\mathcal{F}_{\max \left(N_{1}, \ldots, N_{p}\right)}$ and so $Z$ is in $\mathcal{F}$. So $\mathcal{F}$ is inductive.

From the Zorn lemma, either $\mathcal{F}$ is empty or it admits a maximal element (for the inclusion). If it is empty, then $\mathcal{A}=\mathcal{A}^{\prime}=\emptyset$. If it is not empty and if $\mathcal{F}_{0}:=\bigcap_{m \in \mathcal{C}_{0} \backslash E_{0}} \bar{K}_{m}$ (with $E_{0} \subset \mathcal{C}_{0}$ and $\# E_{0}<\infty$ ) is a maximal element of $\mathcal{F}$, then $\mathcal{A}^{\prime}=\mathcal{F}_{0}$. Indeed, $\mathcal{A}^{\prime}$ contains $\mathcal{F}_{0}$ by definition of $\mathcal{A}^{\prime}$. Conversely, let $S \in \mathcal{A}^{\prime}$, there exists $E \subset \mathcal{C}_{0}$ such that $\# E<\infty$ and such that $S \in \bigcap_{m \in \mathcal{C}_{0} \backslash E} \bar{K}_{m}$. Hence $S \in \bigcap_{m \in \mathcal{C}_{0} \backslash\left(E \cup E_{0}\right)} \bar{K}_{m}$. Since we also have $\bigcap_{m \in \mathcal{C}_{0} \backslash E_{0}} \bar{K}_{m} \subseteq \bigcap_{m \in \mathcal{C}_{0} \backslash\left(E \cup E_{0}\right)} \bar{K}_{m}$, we conclude that $S \in \bigcap_{m \in \mathcal{C}_{0} \backslash E_{0}} \bar{K}_{m}$. Therefore, in any case, $\mathcal{A}$ is contained in an algebraic curve, this gives the $S$-genericity of the birationality of $\rho_{F, S}$ and so the statement of Theorem 2.1 .

\section{Light GENERIC FORMUlas FOR THE DEGREe AND THE Class OF CAUSTICS}

Let $\mathcal{C}=V(F) \subset \mathbb{P}^{2}$ be any irreducible algebraic curve of degree $d \geq 2$. We call isotropic tangent to $\mathcal{C}$ any tangent to $\mathcal{C}$ containing $I$ or $J$. Before stating our formulas, let us introduce some notations. 
For any $P \in \mathbb{P}^{2}$, we write $\mu_{P}(\mathcal{C})$ for the multiplicity of $\mathcal{C}$ at $P$. We recall that $\mu_{P}(\mathcal{C})=1$ means that $P$ is a non singular point of $\mathcal{C}$. For any $P \in \mathcal{C}$, we write $\operatorname{Branch}_{P}(\mathcal{C})$ for the set of branches of $\mathcal{C}$ at $P$. Let us write $\mathcal{E}_{\mathcal{C}}$ for the set of couples $(P, \mathcal{B})$ with $P \in \mathcal{C}$ and with $\mathcal{B} \in \operatorname{Branch}_{P}(\mathcal{C})$. For any $(P, \mathcal{B}) \in \mathcal{E}_{\mathcal{C}}$, we write $\mathcal{T}_{P} \mathcal{B}$ for the tangent line to $\mathcal{B}$ at $P$ and $e_{\mathcal{B}}$ for the multiplicity of $\mathcal{B}$. We recall that $\sum_{\mathcal{B} \in \operatorname{Branch}_{P}(\mathcal{C})} e_{\mathcal{B}}=\mu_{P}(\mathcal{C})$. For any $(P, \mathcal{B}) \in \mathcal{E}_{\mathcal{C}}$ and any algebraic curve $\mathcal{C}^{\prime}$, we denote by $i_{P}\left(\mathcal{C}, \mathcal{C}^{\prime}\right)\left(\right.$ resp. $\left.i_{P}\left(\mathcal{B}, \mathcal{C}^{\prime}\right)\right)$ the intersection number of $\mathcal{C}$ (resp. $\mathcal{B}$ ) with $\mathcal{C}^{\prime}$ at $P$. We recall that the contact number $\Omega_{m_{1}}\left(\mathcal{C}, \mathcal{C}^{\prime}\right)$ of $\mathcal{C}$ with $\mathcal{C}^{\prime}$ is given by $\Omega_{m_{1}}\left(\mathcal{C}, \mathcal{C}^{\prime}\right)=i_{P}\left(\mathcal{C}, \mathcal{C}^{\prime}\right)-\mu_{P}(\mathcal{C}) \mu_{P}\left(\mathcal{C}^{\prime}\right)$. The line at infinity of $\mathbb{P}^{2}$ is written $\ell_{\infty}$. Combining Theorem 2.1 with the main results of $[7,8]$, we obtain:

Proposition 3.1. Let $\mathcal{C}=V(F) \subseteq \mathbb{P}^{2}$ be any irreducible algebraic curve of degree $d \geq 2$ and of class $d^{\vee}$. For a generic $\mathcal{S} \in \mathbb{P}^{2}$, we have

$\operatorname{deg}\left(\Sigma_{S}(\mathcal{C})\right)=3 d+f_{0}-t_{I}-t_{J}$ and $\operatorname{class}\left(\Sigma_{\mathcal{S}}(\mathcal{C})\right)=2 d^{\vee}+d-g-\mu_{I}(\mathcal{C})-\mu_{J}(\mathcal{C})$,

where $g$ is the contact number of $\mathcal{C}$ with $\ell_{\infty}$, i.e. $g:=\sum_{m_{1} \in \mathcal{C} \cap \ell_{\infty}} \Omega_{m_{1}}\left(\mathcal{C}, \ell_{\infty}\right)$, where $f_{0}$ is the number of "inflectional branches" of $\mathcal{C}$ not tangent to the line at infinity, i.e.

$$
f_{0}:=\sum_{(P, \mathcal{B}) \in \mathcal{E}_{\mathcal{C}}: i_{P}\left(\mathcal{B}, \mathcal{T}_{P} \mathcal{B}\right)>2 e_{\mathcal{B}}, \mathcal{T}_{P} \mathcal{B} \neq \ell_{\infty}}\left(i_{P}\left(\mathcal{B}, \mathcal{T}_{P} \mathcal{B}\right)-2 e_{\mathcal{B}}\right) .
$$

and where $t_{P}$ is the number of branches of $\mathcal{C}$ tangent at $P$ to the line at infinity: $t_{P}=\sum_{\mathcal{B} \in \operatorname{Branch}_{P}(\mathcal{C}): \mathcal{T}_{P} \mathcal{B}=\ell_{\infty}} e_{\mathcal{B}}$.

Proof. According to Theorem 2.1, for a generic $S$ in $\mathbb{P}^{2}$, the degree and class (with multiplicity) of $\Sigma_{S}(\mathcal{C})$ are equal to its degree and class. For the degree formula, we use Theorem 20 of [7]. For the class formula, we use Theorem 2 of [8]. For a generic $S \in \mathbb{P}^{2}\left(S \in \mathbb{P}^{2} \backslash\left(\mathcal{C} \cup \ell_{\infty}\right)\right.$ not contained in an isotropic tangent to $\mathcal{C}$ ), we have $f=\mu_{I}(\mathcal{C})+\mu_{J}(\mathcal{C}), f^{\prime}=0, g^{\prime}=0$ and $q^{\prime}=0$ (with the notations of Theorem 2 in [8]).

\section{ACKNOWLEDGEMENTS}

We thank Fabrizio Catanese for discussions having motivated the redaction of this note on source generic results for caustics by reflection.

\section{REFERENCES}

[1] H. Brocard, T. Lemoyne. Courbes géométriques remarquables. Courbes spéciales planes et gauches. Tome I. (French) Nouveau tirage Librairie Scientifique et Technique Albert Blanchard, Paris (1967) viii $+451 \mathrm{pp.}$

[2] F. Catanese and C. Trifogli. Focal loci of algebraic varieties. I. Special issue in honor of Robin Hartshorne. Comm. Algebra 28 (2000), no. 12, pp. 6017-6057.

[3] M. Chasles. Détermination, par le principe des correspondances, de la classe de la développée et de la caustique par réflexion d'une courbe géométrique d'ordre $m$ et de classe $n$ Nouv. Ann. Math. 2 ser. vol. 10 (1871), p. 97-104, extrait C. R. séances A. S. t. LXII.

[4] G. P. Dandelin. Notes sur les caustiques par réflexion, (1822).

[5] B. Fantechi. The Evolute of a Plane Algebraic Curve, (1992) UTM 408, University of Trento.

[6] W. Fulton. Intersection Theory. 2nd edition (1998) Springer Verlag.

[7] A. Josse, F. Pène. On the degree of caustics by reflection. Accepted for publication in Communications in Algebra. 
DEGREE AND CLASS OF CAUSTICS BY REFLECTION FOR A GENERIC SOURCE 5

[8] A. Josse, F. Pène. On the class of caustics by reflection. Preprint. Arxiv 1210.6551.

[9] L. A. J. Quetelet, Énoncés de quelques théorèmes nouveaux sur les caustiques. C. G. Q. (1828) vol 1, p.14, p. 147-149.

[10] G. Salmon G, A treatise on higher plane curves: Intended as a sequel to a treatise on conic sections. Elibron classics (1934).

[11] C. Trifogli. Focal Loci of Algebraic Hypersurfaces: a General Theory, Geom. Dedicata 70 (1998), pp. 1-26.

Université de Brest, LMBA, UMR CNRS 6205, 29238 Brest Cedex, France E-mail address: alfrederic.josse@univ-brest.fr

Université de Brest, LMBA, UMR CNRS 6205, 29238 Brest cedex, France E-mail address: francoise.pene@univ-brest.fr 